\title{
Transseries for the ground state density and generalized Bloch equation: Double-well potential case
}

\author{
E. Shuryak ${ }^{1, *}$ and A. V. Turbiner ${ }^{1,2, \dagger}$ \\ ${ }^{1}$ Department of Physics and Astronomy, Stony Brook University, \\ Stony Brook, New York 11794-3800, USA \\ ${ }^{2}$ Instituto de Ciencias Nucleares, Universidad Nacional Autónoma de México, \\ Apartado Postal 70-543, 04510 México, Distrito Federal, México
}

(Received 2 October 2018; published 16 November 2018)

\begin{abstract}
Based on the generalized Bloch equation, the transseries expansion for the phase (exponent) of the ground state density for double-well potential is constructed. It is shown that the leading and next-toleading terms in semiclassical expansion are still defined by the flucton trajectory (its classical action) and quadratic fluctuations (the determinant), respectively, while the next-to-next-to-leading term (at large distances) is of nonperturbative nature. It comes from the fact that all flucton classical trajectories modified by multi-instanton, instanton-anti-instanton additions lead to the same classical action behavior at large distances. This correction is proportional to sum of all leading instanton contributions to energy gap.
\end{abstract}

DOI: $10.1103 /$ PhysRevD.98.105007

\section{INTRODUCTION}

It was understood long ago that the inter-relations between two formulations of quantum mechanics, Schrödinger's based on the wave functions and Feynman's based on path integrals, become nontrivial in certain special problems. In particular, if coordinates are defined on compact manifolds (such as Lie groups), there exist topologically distinct paths. Since they cannot be continuously deformed into basic topologically trivial paths, the issue of their normalization (and especially their sign) in the path integral formalism is nontrivial and requires basically a separate definition. It has been very clearly explained in the remarkable paper by L. Schulman [1] using the simplest example of a particle on a circle [or $O(2)=U(1)$ group], in which case the question is whether angular momentum should be integer or halfinteger. In the latter case, the wave functions must be defined as antiperiodic, and the winding paths contribution to the integral must be defined as having an extra sign factor. Only with this modification the path integral formalism become finally fixed uniquely.

In our previous works $[2,3]$, we introduced and studied a version of the semiclassical theory based on the so-called flucton paths in Euclidian time, the periodic ones which start

\footnotetext{
*edward.shuryak@stonybrook.edu

turbiner@nucleares.unam.mx, alexander.turbiner@stonybrook .edu

Published by the American Physical Society under the terms of the Creative Commons Attribution 4.0 International license. Further distribution of this work must maintain attribution to the author(s) and the published article's title, journal citation, and DOI. Funded by SCOAP.
}

and end at some arbitrary location $x_{0}$ and thus contribute to the density matrix $\rho\left(x_{0}\right)$. Unlike the textbook WKB (Wentzel-Kramers-Brillouin) approach, this one can be used for multidimensional or Quantum Field Theory (QFT) problems, and perturbative corrections to all orders can be calculated via Feynman diagrams. These corrections has been explicitly calculated, in one and two loops for a number of examples including the quartic anharmonic oscillator and sine-Gordon potential. These series on top of the flucton were then reinterpreted and rederived, using the so-called generalized Bloch equation.

If the potential of the problem has a single minimum, like in anharmonic oscillator $V \sim x^{4}$, the flucton path is uniquely defined by a condition that at the Euclidian time $\tau \rightarrow \pm \infty$ it should "relax" to that minimum. However, if there are two or more degenerate minima (as is the case in the double-well or sin-Gordon problems we also studied), there are also paths which can relax to two different minima. Classical paths, corresponding to transitions between those minima are known as instantons (or anti-instantons, or multi-instantons in general). Contributions of instantons to the ground state energy have been studied in multiple papers, including e.g., our own works $[4,5]$ in which it also has been done explicitly, up to three loops.

The issue we address in this work is the instanton contribution to the density matrix. In Fig. 1, we illustrate it by two paths, both passing through some generic point $x_{0}$ (which we take to be outside of both potential minima marked by wide solid lines). The left sketch shows the flucton path, which at $\tau \rightarrow \pm \infty$ relaxes to the same (nearest) minimum. The right sketch shows a path which relaxes to different minima; we will call it " $\mathrm{f}+\mathrm{i}$ " (flucton plus instanton) path. The Euclidean time $\tau$ is the vertical 


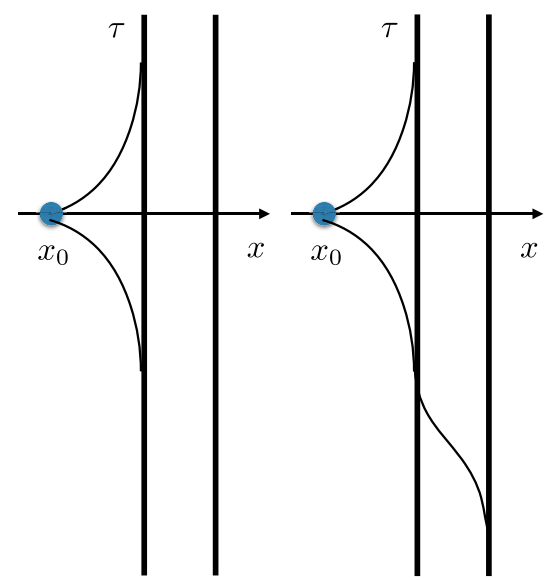

FIG. 1. The flucton path (left) and flucton-plus-instanton path (right) both pass from some generic point $x_{0}$ and relax to one or two degenerate minima, to ensure the finiteness of the action.

coordinate. (Recall that at finite temperatures it is defined on a circle with circumference $\beta=\hbar / T$, and the paths should be periodic. Yet in this work, we consider zero temperature quantum mechanics, so $\beta=\infty$, and the only remaining condition is that the paths must have a finite action.)

Since both paths pass through the point $x_{0}$, they both must contribute to $\rho\left(x_{0}\right)$. Yet since the paths are topologically distinct, the question of relative normalization of their contributions to the integral naturally arises. We already touched upon this issue in our previous paper [5] (for $0<$ $x_{0}<1$ in between the minima), but now we would like to do it more explicitly, using the classic example of the double-well potential and the generalized Bloch equation we also introduced before [5].

Nowadays, it is a well-known fact that in quantum mechanics for potentials with two or more degenerate minima the ground state energy contains nonanalytic terms at $g \rightarrow 0$ of instanton origin in addition to perturbation theory in $g$; see for instance Ref. [6]. In particular, for the ground state of the celebrated quartic double-well potential, the standard perturbation theory expansion for energy becomes transseries of the form,

$$
\begin{aligned}
E\left(g^{2}\right)= & E_{\mathrm{PT}}\left(g^{2}\right)+\sum_{k=1}^{\infty} \sum_{l} \sum_{p=0}^{\infty} \underbrace{\left(\frac{1}{|g|} \exp \left[-\frac{c}{g^{2}}\right]\right)^{k}}_{\text {k-instanton }} \\
& \times\left(\log \frac{c}{g^{2}}\right)^{l} \underbrace{c_{k, l, p} g^{2 p}}_{\mathrm{PT}},
\end{aligned}
$$

see e.g., Ref. [7], in which the parameters $c=1 / 6$ and $c_{k, l, p}$ are real parameters, $g$ is the coupling constant (see below), and the subscript PT stands for perturbation theory. Similar expansion can be derived for all energy eigenvalues. Perhaps, L. D. Landau and E. M. Lifschitz were the first who indicated to this phenomenon [8], J. Zinn-Justin [9] derived this expansion systematically as a state of the art, and together with U. Jentschura [10], they made impressive concrete calculations of this expansion. Recently, G. V. Dunne and M. Ünsal in a number of papers revealed the hidden properties of (1) and made it understandable, at least, for us; see e.g., Ref. [11] and references therein. Note that (1) implies that the energy can be written as sum of perturbative and nonperturbative parts,

$$
E=E_{\mathrm{PT}}+E_{\mathrm{NPT}} .
$$

The aim of this paper is to derive nonanalytic terms in $g$ for ground state density (the square of the ground state function) in a systematic way, thus constructing a type of trans-series for the wave function assuming that the transseries for the ground state energy is known. Explicitly, it is done by separating perturbative and nonperturbative parts in the wave function multiplicatively,

$$
\Psi=e^{-\phi_{\mathrm{PT}}-\phi_{\mathrm{NPT}}} \equiv \psi_{\mathrm{PT}} \psi_{\mathrm{NPT}}
$$

hence, the $\log$ of wave function can be represented as a sum of perturbative and nonperturbative terms. This is the key observation which comes naturally from the Riccati-Bloch equation. Then, we will try to clarify the obtained transseries in the framework of path integral formalism. The celebrated quartic double-well potential will be taken as the example. Thus, overall, the derivation will be made from two different directions: (i) from quantum mechanics using the generalized Bloch equation of the type presented in Ref. [3] and (ii) from the Euclidian time path integral following a variety of flucton-instanton trajectories.

Needless to say, the celebrated quartic double-well potential, written for future convenience in the form

$$
V(x)=\frac{1}{2} x^{2}(1-g x)^{2},
$$

where $g$ is the coupling constant, plays an exceptionally important role in different physical sciences and chemistry. It has two degenerate minima situated at $x=0$ and $x=\frac{1}{g}$, respectively, and a maximum at $x=\frac{1}{2 g}$. The potential is also symmetric with the center of symmetry at $x_{c}=\frac{1}{2 g}$,

$$
V\left(x-\frac{1}{2 g}\right)=V\left(-x+\frac{1}{2 g}\right) .
$$

It is seen explicitly when the potential (4) is rewritten as

$$
V(\tilde{x})=\frac{g^{2}}{2}\left(\tilde{x}-\frac{1}{2 g}\right)^{2}\left(\tilde{x}+\frac{1}{2 g}\right)^{2}-\frac{1}{32 g^{2}},
$$

where $\tilde{x}=x-\frac{1}{2 g}$. It implies the parity of the eigenfunction, being even or odd. Hence, the eigenfunction can be represented in the form

$$
\Psi(x)=\Psi\left(x-\frac{1}{2 g}\right) \pm \Psi\left(-x+\frac{1}{2 g}\right),
$$


with plus sign for even and minus sign for odd eigenfunctions [12]. However, to study the transseries expansion in quantum mechanics for the ground state eigenfunction, it is more convenient to use the exponential representation (3) where the phase is given by the sum of perturbative and nonperturbative parts,

$$
\log \Psi=\log \Psi_{\mathrm{PT}}+\log \Psi_{\mathrm{NPT}} .
$$

In QFT and quantum mechanics in the path integral formalism the density matrix, calculated in saddle-point method, appears as the sum over saddle point contributions as for large positive (negative) distance $x_{0}$, hence, in the form of a linear superposition. It implies that the representation (6) is more natural than non-linear representation (3). However, the concrete calculations are performed much more easily in the representation (3). Afterwards the results can be re-expanded (in additive way) for the representation (6), see Sec. IV.

The potential (4) belongs to a special class of anharmonic potentials,

$$
V(x)=\frac{\tilde{V}(g x)}{g^{2}}=\frac{1}{2} x^{2}+a_{3} g x^{3}+a_{4} g^{2} x^{4}+\ldots,
$$

as well as the celebrated sine-Gordon potential. The function $\tilde{V}$ has a minimum at $x=0$ it always starts from the quadratic term. The frequency of the small oscillations in the potential $V$ near the minimum can always be placed equal to $1, \omega=1$ and $g$ is the coupling constant of dimension $\left[\frac{1}{x}\right.$ ], see e.g., Ref. [3]. For the sake of future convenience, the classical (vacuum) energy is always taken to be zero, $V(0)=0$, and $a_{2,3, \ldots}$ are real, dimensionless parameters; hence, $V(x) \geq 0$. We call $(g x)$ the classical coordinate; see below. Both the classical coordinate and the Hamiltonian with the potential (7),

$$
\begin{aligned}
& \mathcal{H}=-\frac{1}{2 m} \partial_{x}^{2}+\frac{1}{g^{2}} \tilde{V}(g x), \quad \partial_{x}=\frac{d}{d x}, \\
& m=1, \quad x \in(-\infty, \infty),
\end{aligned}
$$

are invariant with respect to simultaneous change,

$$
x \rightarrow-x, \quad g \rightarrow-g .
$$

It implies that the energy is the function of $g^{2}$,

$$
E=E\left(g^{2}\right) .
$$

A particular form of the transseries (1) for the ground state energy of the quartic double-well potential (4), which we are going to exploit, has the form (if for the sake of simplicity we assume $g>0$ )

$$
\begin{aligned}
E(g)= & E_{\mathrm{PT}}+E_{\mathrm{NPT}}=\sum_{n=0} g^{2 n} E_{\mathrm{PT}, n}+\frac{1}{g} e^{-S_{0}}\left(A_{0}^{(1)}+A_{1}^{(1)} g^{2}+\ldots\right)+\frac{1}{g^{2}} e^{-2 S_{0}}\left(A_{0}^{(2)}+A_{1}^{(2)} g^{2}+\ldots\right) \\
& +\ldots+\log \left(g^{2}\right) \frac{1}{g} e^{-S_{0}}\left(\frac{1}{g} e^{-S_{0}}\left(B_{0}^{(1)}+B_{0,1}^{(1)} g^{2}+\ldots\right)+\frac{1}{g^{2}} e^{-2 S_{0}}\left(B_{1}^{(1)}+B_{1,1}^{(1)} g^{2}+\ldots\right)+\ldots\right) \\
& +\log ^{2}\left(g^{2}\right) \frac{1}{g^{2}} e^{-2 S_{0}}\left(\frac{1}{g} e^{-S_{0}}\left(B_{0}^{(2)}+B_{0,1}^{(2)} g^{2}+\ldots\right)+\frac{1}{g^{2}} e^{-2 S_{0}}\left(B_{1}^{(2)}+B_{1,1}^{(2)} g^{2}+\ldots\right)+\ldots\right)+\ldots
\end{aligned}
$$

where $S_{0}=\frac{1}{6 q^{2}}$ is one-instanton classical action; the parameters $E \mathrm{~s}, A \mathrm{~s}$, and $B \mathrm{~s}$ are real and can be calculated constructively; and some of them are explicitly known (see Ref. [10] and references therein). The form (10) is slightly different from the standard form of transseries, see e.g., Ref. [11], being of the type (1): it takes into account the appearance in the standard form for transseries the imaginary parts in some coefficients with their further cancellations due to the Bogomolny mechanism [13,14]. It is worth emphasizing that one can see explicitly in (10) the presence of two structures,

$$
\xi=\frac{1}{g} e^{-S_{0}}, \quad \chi=\log \left(g^{2}\right) \frac{1}{g} e^{-S_{0}},
$$

in addition to the coupling constant $g$ itself, cf. Ref. [10], Eqs. (8.1) and (8.2). Therefore, the transseries (10) can be considered as the triple Taylor expansion in $g, \xi, \chi$,

$$
E=\sum E_{k, \ell, p} g^{2 k} \xi^{\ell} \chi^{p}
$$

Note that $\chi$ has the meaning of one-instantion contribution in a leading order: classical action plus determinant. It is worth noting that nonperturbative energy $E_{\mathrm{NPT}}$ can be reorganized into the form of perturbation series,

$$
E_{\mathrm{NPT}}=\sum_{n=0} g^{2 n} A_{n} E_{\mathrm{NPT}, n}(g),
$$

where

$$
\begin{aligned}
E_{\mathrm{NPT}, 0}= & \left\{\frac{1}{g} e^{-S_{0}}+\frac{1}{g^{2}} e^{-2 S_{0}}\left[A_{0}^{(2)}+B_{0}^{(2)} \log \left(g^{2}\right)\right]\right. \\
& +\frac{1}{g^{3}} e^{-3 S_{0}}\left[A_{0}^{(3)}+B_{0,1}^{(3)} \log \left(g^{2}\right)+B_{0,2}^{(3)} \log ^{2}\left(g^{2}\right)\right] \\
& \left.+\ldots+\frac{1}{g^{p}} e^{-p S_{0}} \sum_{q=0}^{p-1} A_{0, q}^{(p)} \log ^{q}\left(g^{2}\right)+\ldots\right\}
\end{aligned}
$$


with $A_{0}=-\sqrt{\frac{1}{\pi}}$ in (13). This is the sum of all leading instanton contributions to nonperturbative energy. In fact, it represents the sum over multi-instanton saddle points in the leading approximation, when classical action plus determinant (one-loop contribution) are taken into account. The $n$th correction in (13) has a similar form:

$$
\begin{aligned}
E_{\mathrm{NPT}, \mathrm{n}}= & \left\{\frac{1}{g} e^{-S_{0}}+\frac{1}{g^{2}} e^{-2 S_{0}}\left[A_{n}^{(2)}+B_{n}^{(2)} \log \left(g^{2}\right)\right]\right. \\
& +\frac{1}{g^{3}} e^{-3 S_{0}}\left[A_{n}^{(3)}+B_{n, 1}^{(3)} \log \left(g^{2}\right)+B_{n, 2}^{(3)} \log ^{2}\left(g^{2}\right)\right] \\
& \left.+\ldots+\frac{1}{g^{p}} e^{-p S_{0}} \sum_{q=0}^{p-1} A_{n, q}^{(p)} \log ^{q}\left(g^{2}\right)+\ldots\right\} .
\end{aligned}
$$

Natural questions to ask are whether transseries expansion exists for a wave function of the type (12) with $x$-dependent coefficients and if so how to construct it. In order to proceed, let us derive the generalized Bloch equation, cf. Ref. [3], specific for the potential with two degenerate minima. The first step is standard: we begin with the Schrödinger equation for the wave function and go to the equation on its logarithmic derivative $y(x)$, which eliminates the overall normalization constant from consideration. We arrive at the familiar Riccati equation where the boundary condition $y(0)=0$ should be imposed. However, in order to find the non-singular at real $x$ solution which will guarantee the normalizability of the eigenfunction, two extra conditions should be imposed: (i) $y$ should be asymptotically antisymmetric, $y(-x)=-y(x)$; in concrete, it should behaves asymptotically like $y(x) \sim g x|x|$ at large $|x|$ (at $g>0$ ), and (ii) derivative at origin is equal to the eigenvalue, $y^{\prime}(0)=E$. The condition (ii) reveals the meaning of the quantization of energy in the nonlinear Riccati equation: for given $g$, there exists the single value of the ground state energy $E(g)$ for which (i) holds. The second step is that we have to extract the product of two linear functions of the coordinate from the logarithmic derivative assuming the remaining function depends essentially on the classical coordinate $(g x)$,

$$
x(1-g x) z(g x, g)=-\frac{\psi^{\prime}(x)}{\psi(x)}=y(x) .
$$

It reflects the fact that, since the original potential $V(x)(7)$ has two minima at $x=0$ and $x=1 / g$, the logarithmic derivative of the wave function (the derivative of the phase) has to vanish linearly at $x=0$ and $x=1 / g$, respectively. Now, we have to write the equation for function $z$. Substituting the construction (16) to the Schrödinger equation

$$
\left(-\frac{1}{2} \frac{d^{2}}{d x^{2}}+\frac{1}{g^{2}} \tilde{V}(g x)\right) \psi(x)=E \psi(x)
$$

where the Planck constant is placed equal to $1, \hbar=1$, and redefining the coordinate $u=g x$ assuming $g>0$, we arrive at the equation

$$
\begin{gathered}
g^{2} u(1-u) z^{\prime}(u)+g^{2}(1-2 u) z(u)-u^{2}(1-u)^{2} z(u)^{2} \\
=2 g^{2} E-\tilde{V}(u), \quad \tilde{V}(u)=u^{2}(1-u)^{2},
\end{gathered}
$$

which is called the generalized Bloch equation. Note that here $z(u)$ has the meaning of a reduced logarithmic derivative; see (16). We will study Eq. (17), imposing the boundary condition $z(0)=E$ and putting also the condition $z(u) \sim \mp 1$ at $u \rightarrow \pm \infty$.

Now, we proceed in solving Eq. (17) at the weak coupling regime $g \rightarrow 0$ by expanding consistently both the energy $E$ and $z(u)$ in transseries (10) and

$$
\begin{aligned}
z(u)= & \sum_{n=0} g^{2 n} z_{\mathrm{PT}, n}(u)+g e^{-S_{0}}\left(\zeta_{0}^{(1)}(u)+g^{2} \zeta_{1}^{(1)}(u)+\ldots\right)+e^{-2 S_{0}}\left(\zeta_{0}^{(2)}(u)+g^{2} \zeta_{1}^{(2)}(u)+\ldots\right) \\
& +\ldots+\log g^{2}\left(e^{-2 S_{0}}\left(\tilde{\zeta}_{0}^{(2)}(u)+g^{2} \tilde{\zeta}_{1}^{(2)}(u)+\ldots\right)+\frac{1}{g} e^{-3 S_{0}}\left(\tilde{\zeta}_{0}^{(3)}(u)+g^{2} \tilde{\zeta}_{1}^{(3)}(u)+\ldots\right)+\ldots\right) \\
& +\frac{1}{g} \log ^{2} g^{2}\left(e^{-3 S_{0}}\left(\hat{\zeta}_{0}^{(3)}+g^{2} \hat{\zeta}_{1}^{(3)}+\ldots\right)+\frac{1}{g^{2}} e^{-4 S_{0}}\left(\hat{\zeta}_{0}^{(4)}+g^{2} \hat{\zeta}_{1}^{(4)}+\ldots\right)+\ldots\right)+\ldots,
\end{aligned}
$$

respectively. We will explore in details the following issues: (i) the perturbation theory in powers of $g$, (ii) the one-instanton contribution $\sim e^{-S_{0}}$, (iii) two-instanton contributions $\sim e^{-2 S_{0}}$, and (iv) the sum of leading multi-instanton contributions.

\section{WEAK COUPLING REGIME: PERTURBATION SERIES VS SEMICLASSICAL EXPANSION}

Looking at the generalized Bloch equation (17), one can immediately realize a striking fact that the perturbation theory expansion 


$$
\begin{aligned}
& E_{\mathrm{PT}}(g)=\sum_{n=0} g^{2 n} E_{\mathrm{PT}, n}, \\
& z_{\mathrm{PT}}(u)=\sum_{n=0} g^{2 n} z_{\mathrm{PT}, n}(u)
\end{aligned}
$$

can be constructed self-consistently, without involving nonperturbative, exponentially small terms, cf. Ref. [3], Sec. III.C.2. Owing to this property, we can separate perturbative and nonperturbative contributions in $z$. From now on, we will drop the notation "PT" in $z$ but will keep it for energy $E$.

In the zeroth order in $g, O\left(g^{0}\right)$ in (17), in which all terms proportional to the coupling are ignored, the equation to solve is very simple,

$$
-u^{2}(1-u)^{2} z_{0}(u)^{2}=-u^{2}(1-u)^{2},
$$

leading to

$$
z_{0}(u)= \pm 1
$$

here, the sign is chosen by requiring the normalizability of the unperturbed wave function $\Psi_{0}$. Thus, we take the sign plus for $u<0: z_{0}=-1$ and the sign minus for $u>0$ : $z_{0}=1$. Hence, the solution is discontinuous at $u=0$. This is the indication that we cannot go to the domain of small $|u|$ : the expansion (19) for $z_{\mathrm{PT}}$ is convergent at large $|u|>$ $u_{0}$ only, see below.

This result $\left[u(1-u) z_{0}\right]$ is, in fact, the classical momentum at zero energy, and therefore, when we return to the wave function, the zeroth order term gives the wellknown semiclassical action. So, the zero approximation admits a simple interpretation as the exponent is equal to the classical action in the semiclassical wave function $\psi \sim \exp \left(-\int{ }^{x} p\left(x^{\prime}\right) d x^{\prime}\right)$ but at zero energy.

Moving to the next term $z_{P T, 1}=z_{1}$ of the expansion (19), one finds the following equation $O\left(g^{2}\right)$ in order to find it:

$$
\begin{aligned}
& u(1-u) z_{0}^{\prime}(u)+(1-2 u) z_{0}(u)-2 u^{2}(1-u)^{2} z_{0}(u) z_{1}(u) \\
& \quad=2 E_{\mathrm{PT}, 0} .
\end{aligned}
$$

Note here that the equation involves the known function $z_{0}$ and unknown $z_{1}$, and both of them appear linearly. The similar feature takes place in all orders; finding $z_{n}$ does not involve solving a differential equation rather than a linear algebraic one.

An important feature of the procedure is that the perturbative energy $E_{\mathrm{PT}}$ needs to be used in (17) instead of $E$, in the form of perturbative expansion in powers of $g^{2}$. These coefficients $E_{\mathrm{PT}, n}$ should be found separately, by some other method, not via the perturbation theory in the generalized Bloch equation. For example, the nonlinearization procedure can be used for it [15]. Since the zeroth order potential is the harmonic oscillator one, $E_{\mathrm{PT}, 0}=1 / 2$. Hence, the first correction, which emerges from (22), is given by

$$
z_{1}(u)=\frac{(1-2 u) z_{0}-1}{2 u^{2}(1-u)^{2} z_{0}}
$$

which is a rational function in $u$. At large $u>0$, the correction tends to zero, $z_{1} \rightarrow-u^{-3}$, in agreement with boundary conditions at large $|u|$. Otherwise, it grows up to infinity with decreasing $|u|$ toward 0 or 1 . It implies that we cannot go to the domain of small $|u|$ and should remain at large $|u|$, which is typical for the semiclassical approximation. In Ref. [3], it was shown explicitly that this correction is related to the determinant in flucton loop expansion. In a similar way, one can find $z_{2}(u)$ using the first perturbation correction $E_{\mathrm{PT}, 1}$ and known $z_{0,1}$ by solving the equation

$$
\begin{aligned}
& u(1-u) z_{1}^{\prime}(u)+(1-2 u) z_{1}(u)-u^{2}(1-u)^{2} \\
& \quad \times\left(z_{1}^{2}+2 z_{0}(u) z_{2}(u)\right)=2 E_{\mathrm{PT}, 1} .
\end{aligned}
$$

As a result,

$z_{2}(u)=\frac{u(1-u) z_{1}^{\prime}(u)+(1-2 u) z_{1}-2 E_{\mathrm{PT}, 1}}{2 u^{2}(1-u)^{2} z_{0}}-\frac{z_{1}^{2}}{2 z_{0}}$

is the rational function in $u$. At $|u| \rightarrow \infty, z_{2} \sim-\frac{3}{2 u^{6}}$, overall, it is of the order $O\left(g^{4}\right)$. This correction is related with the twoloop contribution in flucton loop expansion [3].

In general, in the same way, one can write the equation for $z_{n}(u)$,

$$
\begin{aligned}
& u(1-u) z_{n-1}^{\prime}(u)+(1-2 u) z_{n-1}(u)-u^{2}(1-u)^{2} \\
& \quad \times\left[Q_{n}+2 z_{0}(u) z_{n}(u)\right]=2 E_{\mathrm{PT}, n-1},
\end{aligned}
$$

where

$$
Q_{n}=\sum_{i=1}^{n-1} z_{i} z_{n-i} .
$$

Finally, the solution gets the form

$z_{n}(u)=\frac{u(1-u) z_{n-1}^{\prime}(u)+(1-2 u) z_{n-1}-2 E_{\mathrm{PT}, n-1}}{2 u^{2}(1-u)^{2} z_{0}}-\frac{Q_{n}}{2 z_{0}}$.

In general, it is the rational function in $u$,

$$
z_{n}(u)=\frac{p_{n}(u)}{2 u^{2 n}(1-u)^{2 n} z_{0}^{n}}=\frac{p_{n}(u)}{2 \tilde{V}^{n}},
$$

where $p_{n}$ is the $n$th degree polynomial with rational coefficients and $\tilde{V}$ is the potential defined (17). Thus, $z_{n}(u)$ is given by the sum of $n$-loop Feynman diagrams weighted with appropriate symmetry factors in flucton calculus. 


\section{WEAK COUPLING REGIME: TRANSSERIES EXPANSION, EXPONENTIALLY SMALL TERMS}

\section{A. One-instanton contribution}

Analyzing the generalized Bloch equation (17), one can immediately realize the striking fact that the one-instanton contribution to energy and reduced logarithmic derivative

$$
\begin{aligned}
& E_{1 I}(g)=A_{0} e^{-S_{0}} \sum_{n=0} g^{2 n-1} E_{1 I, n}, \\
& z_{1 I}(u)=A_{0} e^{-S_{0}} \sum_{n=0} g^{2 n+1} \zeta_{n}^{(1)}(u),
\end{aligned}
$$

see (10), can be constructed without involving exponentially small terms of higher orders $e^{-p S_{0}}, p=2$. Here $A_{0}=-\sqrt{\frac{1}{\pi}}$ is normalization factor given by the instanton determinant at $g=1$ and $E_{1 I, n}$ define energy corrections to one-instanton; systematically, they are rational numbers $E_{1 I, 0}=1, \quad E_{1 I, 1}=-\frac{71}{12}, \quad E_{1 I, 2}=-\frac{6299}{288} \ldots \quad[10], \quad$ Sec. 8, Eq. (8.13a). Note that $E_{1 I, n}$ at $n=1,2$ were calculated alternatively in instanton calculus using two- and threeloop Feynman integrals $[4,16]$, respectively.

Now, we proceed to the calculation of exponentially small terms in $g$ in expansion (18), (29). For the first step, let us collect all terms of the order $O\left(g e^{-S_{0}}\right)$ in Eq. (17), which is of the lowest order in $g$ in front of the exponentially small term $e^{-S_{0}}$,

$$
-2 u^{2}(1-u)^{2} z_{0}(u) \zeta_{0}^{(1)}(u)=-2 E_{1 I, 0},
$$

cf. Eq. (20), where $E_{1 I, 0}=1$, see e.g., Ref. [9], and $z_{0}$ is given by (21). Its solution has the form

$$
\zeta_{0}^{(1)}=\frac{1}{u^{2}(1-u)^{2} z_{0}}=\frac{1}{\tilde{V} z_{0}},
$$

for $|u|>1$, and here the potential $\tilde{V}$ is defined in (17). Asymptotically,

$$
\zeta_{0}^{(1)} \rightarrow \frac{1}{z_{0} u^{4}}, \quad u \rightarrow \pm \infty
$$

hence, the boundary condition at $u= \pm \infty$ is satisfied. For the next step, let us collect all terms of the order $O\left(g^{3} e^{-S_{0}}\right)$ in Eq. (17), which is of the next-to-lowest order in $g$ in front of the exponentially small term $e^{-S_{0}}$,

$$
\begin{aligned}
u(1-u)\left(\partial_{u} \zeta_{0}^{(1)}(u)\right)+(1-2 u) \zeta_{0}^{(1)}(u) \\
\quad-2 u^{2}(1-u)^{2} z_{0} \zeta_{1}^{(1)}(u) \\
=-2 E_{1 I, 1}+2 u^{2}(1-u)^{2} z_{1} \zeta_{0}^{(1)}(u),
\end{aligned}
$$

where $E_{1 I, 1}=-71 / 12$, see e.g., Ref. [9] and also Ref. [16], and $z_{1}$ is given by (23). Its solution has the form

$$
\begin{aligned}
\zeta_{1}^{(1)}= & \frac{1}{z_{0}}\left(-\frac{71}{12 u^{2}(1-u)^{2}}-z_{1} \zeta_{0}^{(1)}+\frac{\partial_{u} \zeta_{0}^{(1)}}{2 u(1-u)}\right. \\
& \left.+\frac{(1-2 u) \zeta_{0}^{(1)}}{2 u^{2}(1-u)^{2}}\right)
\end{aligned}
$$

In general, collecting terms of the order $O\left(g^{2 n+1} e^{-S_{0}}\right)$ in Eq. (17), we arrive at the equation

$$
\begin{aligned}
u( & -u)\left(\partial_{u} \zeta_{n-1}^{(1)}(u)\right)+(1-2 u) \zeta_{n-1}^{(1)}(u) \\
& -2 u^{2}(1-u)^{2} z_{0} \zeta_{n}^{(1)}(u) \\
= & -2 E_{1 I, n}+2 u^{2}(1-u)^{2} Q_{n}^{(1)}
\end{aligned}
$$

where

$$
Q_{n}^{(1)}=\sum_{i=1}^{n} z_{i}(u) \zeta_{n-i}^{(1)}(u)
$$

It is easily solved, and the explicit form of the $n$th correction reads

$$
\begin{aligned}
\zeta_{n}^{(1)}= & \frac{1}{z_{0}}\left(-\frac{E_{1 I, n}}{u^{2}(1-u)^{2}}-Q_{n}^{(1)}+\frac{\partial_{u} \zeta_{n-1}^{(1)}}{2 u(1-u)}\right. \\
& \left.+\frac{(1-2 u) \zeta_{n-1}^{(1)}}{2 u^{2}(1-u)^{2}}\right) .
\end{aligned}
$$

Finally, the $n$th correction has the form of a rational function with integer coefficients similar to (28).

Concluding, one can see that in order to construct $z_{1 I}(u)$ we have to know the perturbative contribution $z_{\mathrm{PT}}(u)$ only. It is a type of nested construction.

\section{B. Two-instanton contribution}

From the generalized Bloch equation (17), one can immediately realize that the two-instanton contribution

$$
\begin{aligned}
& E_{2 I}(g)=A_{0}^{(2)} e^{-2 S_{0}} \sum_{n=0} g^{2 n-2} E_{2 I, n}, \\
& z_{2 I}(u)=A_{0}^{(2)} e^{-2 S_{0}} \sum_{n=0} g^{2 n} \zeta_{n}^{(2)}(u),
\end{aligned}
$$

see (10), second line, and (18), second line, can be constructed without involving exponentially small terms of higher orders $e^{-p S_{0}}, p>2$ or logarithmic contributions $\log ^{q}\left(g^{2}\right) e^{-p S_{0}}, q \geq 1, p \geq 2$.

Here, $A_{0}^{(2)}=\frac{1}{\pi}$ is a normalization factor given seemingly by the two-instanton determinant at $g=1$ and $E_{2 I, n}$ define energy corrections to two-instanton. Systematically, they are written in the form of linear function in Euler constant $\gamma$ with rational coefficients: 


$$
\begin{aligned}
& E_{2 I, 0}=\gamma, \quad E_{2 I, 1}=-\frac{23}{2}-\frac{53}{6} \gamma, \\
& E_{2 I, 2}=\frac{13}{12}-\frac{1277}{72} \gamma \ldots ;
\end{aligned}
$$

see [10], Sec. 8, Eq. (8.14a). We are not familiar with any attempt to calculate these coefficients in instanton calculus using the Feynman diagramm techniques.

Collecting the terms of the order $O\left(g^{0} e^{-2 S_{0}}\right)$ in Eq. (17), which is the lowest order in $g$ in front of the exponentially small term $e^{-2 S_{0}}$, we arrive at

$$
-2 u^{2}(1-u)^{2} z_{0}(u) \zeta_{0}^{(2)}(u)=-2 E_{2 I, 0},
$$

cf. Eq. (20), where $E_{2 I, 0}=1$, see e.g., Ref. [9], and $z_{0}$ is given by (21). Its solution has the form

$$
\zeta_{0}^{(2)}=\frac{1}{u^{2}(1-u)^{2} z_{0}}=\frac{1}{\tilde{V} z_{0}},
$$

for $|u|>1$; here, the potential $\tilde{V}$ is defined at (17). It coincides with $\zeta_{0}^{(1)}(31)$.

For the next step, let us collect all terms of the order $O\left(g^{2} e^{-2 S_{0}}\right)$ in Eq. (17), which is of the next-to-lowest order in $g$ in front of the exponentially small term $e^{-2 S_{0}}$,

$$
\begin{aligned}
u(1-u)\left(\partial_{u} \zeta_{0}^{(2)}(u)\right)+(1-2 u) \zeta_{0}^{(2)}(u) \\
\quad-2 u^{2}(1-u)^{2} z_{0} \zeta_{1}^{(2)}(u) \\
=-2 E_{2 I, 1}+u^{2}(1-u)^{2}\left(2 z_{1} \zeta_{0}^{(2)}+\left(\zeta_{0}^{(1)}\right)^{2}\right),
\end{aligned}
$$

where $z_{1}$ is given by (23) and $\zeta_{0}^{(1)}$ is from (31). Its solution has the form

$$
\begin{aligned}
\zeta_{1}^{(2)}= & \frac{1}{z_{0}}\left(\frac{E_{2 I, 1}}{u^{2}(1-u)^{2}}-\frac{1}{2}\left(2 z_{1} \zeta_{0}^{(2)}+\left(\zeta_{0}^{(1)}\right)^{2}\right)\right. \\
& \left.+\frac{\partial_{u} \zeta_{0}^{(2)}}{2 u(1-u)}+\frac{(1-2 u) \zeta_{0}^{(2)}}{2 u^{2}(1-u)^{2}}\right)
\end{aligned}
$$

It is easy to find the $n$th correction,

$$
\zeta_{n}^{(2)}=\frac{1}{z_{0}}\left(\frac{E_{2 I, n}}{u^{2}(1-u)^{2}}-\frac{Q_{n}^{(2)}}{2}+\frac{\partial_{u} \zeta_{n-1}^{(2)}}{2 u(1-u)}+\frac{(1-2 u) \zeta_{n-1}^{(2)}}{2 u^{2}(1-u)^{2}}\right),
$$

where

$$
Q_{n}^{(2)}=2 \sum_{i=1}^{n} z_{i}(u) \zeta_{n-i}^{(2)}(u)+\sum_{i=0}^{n-1} \zeta_{i}^{(1)}(u) \zeta_{n-i}^{(1)}(u) .
$$

It is evident that in order to construct two-instanton contribution $z_{2 I}(u)$ we have to know perturbative contribution $z_{\mathrm{PT}}(u)$ and one-instanton contribution $z_{1 I}(u)$ only. As a result, the correction $\zeta_{n}^{(2)}$ is a rational function.

It is needless to demonstrate that in order to determine the $k$-instanton contribution,

$$
\begin{aligned}
& E_{k I}(g)=A_{0}^{(k)} e^{-2 S_{0}} \sum_{n=0} g^{2 n-k} E_{2 I, n}, \\
& z_{k I}(u)=A_{0}^{(k)} e^{-2 S_{0}} \sum_{n=0} g^{2 n-k+2} \zeta_{n}^{(2)}(u),
\end{aligned}
$$

we have to know perturbative contribution $z_{\mathrm{PT}}(u)$ and all one-, two-, and $(k-1)$-instanton contributions $z_{(k-1) I}(u)$. It is a type of nested construction, and it does not involve logarithmic contributions.

\section{Two-instanton log contribution}

From the generalized Bloch equation (17), one can immediately realize that the two-instanton contribution

$$
\begin{aligned}
E_{2 I-\log }(g) & =A_{0}^{(2 l)} \log \left(g^{2}\right) e^{-2 S_{0}} \sum_{n=0} g^{2 n-2} E_{2 I l, n}, \\
z_{2 I l}(u) & =A_{0}^{(2 l)} \log \left(g^{2}\right) e^{-2 S_{0}} \sum_{n=0} g^{2 n} \zeta_{n}^{(2 l)}(u),
\end{aligned}
$$

see (10), second line, and see (18), second line, can be constructed without involving exponentially small terms of higher orders $e^{-p S_{0}}, p>2$ or logarithmic contributions $\log ^{q}\left(g^{2}\right) e^{-p S_{0}}, q \geq 1, p>2$.

Here, $A_{0}^{(2 l)}=\frac{1}{\pi}$ is the normalization factor given seemingly by the two-instanton determinant at $g=1$ and $E_{2 I l, n}$ define energy corrections to two-instanton logarithmic contribution, systematically, they are given by rational coefficients:

$$
E_{2 I l, 0}=1, \quad E_{2 I l, 1}=-\frac{53}{6}, \quad E_{2 I l, 2}=\frac{1277}{72}, \ldots ;
$$

see Ref. [10], Sec. 8, Eq. (8.14a). We are not familiar with any attempt to calculate these coefficients in instanton calculus.

Collecting the terms of the order $O\left(\log \left(g^{2}\right) e^{-2 S_{0}}\right)$ in Eq. (17), which is the lowest order in $g$ in front of the exponentially small term $\log \left(g^{2}\right) e^{-2 S_{0}}$, we arrive at

$$
-2 u^{2}(1-u)^{2} z_{0}(u) \zeta_{0}^{(2 l)}(u)=-2 E_{2 I l, 0},
$$

cf. Eq. (20), where $E_{2 I, 0}=1$, see e.g., Ref. [9], and $z_{0}$ is given by (21). Its solution has the form

$$
\zeta_{0}^{(2 l)}=\frac{1}{u^{2}(1-u)^{2} z_{0}}=\frac{1}{\tilde{V} z_{0}}
$$

for $|u|>1$; here, the potential $\tilde{V}$ is defined at (17). It coincides with $\zeta_{0}^{(1)}$ (31) and with $\zeta_{0}^{(2)}$ (39). 
It is easy to find the $n$th correction,

$$
\zeta_{n}^{(2 l)}=\frac{1}{z_{0}}\left(\frac{E_{2 I, n}}{u^{2}(1-u)^{2}}-\frac{Q_{n}^{(2 l)}}{2}+\frac{\partial_{u} \zeta_{n-1}^{(2 l)}}{2 u(1-u)}+\frac{(1-2 u) \zeta_{n-1}^{(2 l)}}{2 u^{2}(1-u)^{2}}\right),
$$

where

$$
Q_{n}^{(2 l)}=2 \sum_{i=1}^{n} z_{i}(u) \zeta_{n-i}^{(2)}(u) .
$$

One can see that in order to construct $\zeta_{2 I l}(u)$ we have to know perturbative contribution $z_{\mathrm{PT}}(u)$ only. Thus, it is a type of nested construction. As a result, the correction $\zeta_{n}^{(2 l)}$ is a rational function.

\section{Leading semiclassical multi-instanton-inspired correction}

The sum of the exponentially small contributions to the ground state energy in the leading order, when the perturbation theory around the multi-instanton is neglected, can be written in the form

$$
\begin{aligned}
A_{0} E_{\mathrm{NPT}, 0}= & A_{0}\left(\sum_{p=1} B_{0}^{\left(l^{0}, p\right)} g^{-p} e^{-p S_{0}}+\log \left(g^{2}\right)\right. \\
& \times \sum_{p=2} B_{0}^{(l, p)} g^{-p} e^{-p S_{0}}+\log ^{2}\left(g^{2}\right) \\
& \left.\times \sum_{p=3} B_{0}^{\left(l^{2}, p\right)} g^{-p} e^{-p S_{0}}+\ldots\right),
\end{aligned}
$$

cf. Eq. (14). We assume and then check correctness afterward that the sum of the exponentially small contributions in $g$ to the reduced phase $z$ in the leading order, when the perturbation theory around multi-instanton is neglected, has the form

$$
\begin{aligned}
A_{0} \zeta_{\mathrm{NPT}, 0}(u) & =A_{0}\left(\sum_{p=1} B_{0}^{\left(l^{0}, p\right)} g^{-p+2} e^{-p S_{0}} \zeta_{0}^{\left(l^{0}, p\right)}(u)\right. \\
& +\log \left(g^{2}\right) \sum_{p=2} B_{0}^{(l, p)} g^{-p+2} e^{-p S_{0}} \zeta_{0}^{(l, p)}(u) \\
& \left.+\log ^{2}\left(g^{2}\right) \sum_{p=3} B_{0}^{\left(l^{2}, p\right)} g^{-p+2} e^{-p S_{0}} \zeta_{0}^{\left(l^{2}, p\right)}(u)+\ldots\right),
\end{aligned}
$$

where $l^{q}$ in the superscript of $B_{0}^{\left(l^{2}, p\right)}$ means presence of the $\log ^{q}$ in front of the sum.

Now, let us take the generalized Bloch equation (17), substitute in there the energy in the form (10) and the reduced logarithmic derivative $z(u, g)$ in the form (18), and collect carefully, one by one, the expressions in $g$ and $e^{-S_{0}}$ which occur in (49). Finally, it turns out that the coefficient in front of the defining expression has the form

$$
-2 u^{2}(1-u)^{2} z_{0}(u) \zeta_{0}(u)+2 B_{0}=0
$$

(where upper indices in $\zeta_{0}$ and $B_{0}$ are dropped for convenience), independently on upper indices, cf. Eq. (20) as well as (30), (38), and (45); here, $z_{0}$ is given by (21). Its solution has the form

$$
\zeta_{0}(u)=\frac{B_{0}}{u^{2}(1-u)^{2} z_{0}}=\frac{B_{0}}{\tilde{V} z_{0}},
$$

for $|u|>1$; here, the potential $\tilde{V}$ is defined at (17). Substituting (51) into (49), we arrive at an unexpectedly compact expression,

$$
\zeta_{\mathrm{NPT}, 0}(u)=\frac{E_{\mathrm{NPT}, 0}}{u^{2}(1-u)^{2} z_{0}}=\frac{E_{\mathrm{NPT}, 0}}{\tilde{V}(u) z_{0}} .
$$

It corresponds to logarithmic derivative

$$
y_{\mathrm{NPT}, 0}=x(1-g x) \zeta_{\mathrm{NPT}, 0}(g x)=\frac{1}{g^{2}} \frac{E_{\mathrm{NPT}, 0}}{\sqrt{V(x))} z_{0}},
$$

and the nonperturbative phase at large $x \gg 0$ is equal to

$$
\begin{aligned}
\phi_{\mathrm{NPT}, 0}(x) & =\frac{E_{\mathrm{NPT}, 0}}{g^{2} z_{0}} \int \frac{1}{\sqrt{V(x)}} d x=-\frac{E_{\mathrm{NPT}, 0}}{g^{2}} \log \left(1-\frac{1}{g x}\right) \\
& \approx \frac{E_{\mathrm{NPT}, 0}}{g^{2}} \frac{1}{g x} .
\end{aligned}
$$

Hence, the leading nonperturbative contribution to phase in semiclassical domain is subdominant in comparison to both the classical action, which is the leading (dominant) contribution,

$$
\phi_{\mathrm{PT}, 0}(x)(x)=g \frac{x^{3}}{3}-\frac{x^{2}}{2}, \quad x \gg 0,
$$

also the first perturbative correction, which is next-to-leading contribution

$$
\phi_{\mathrm{PT}, 1}(x)(x)=\log g x ;
$$

see (23). However, the second perturbative correction (25), which leads to the next-to-next-to-leading contribution,

$$
\phi_{\mathrm{PT}, 2}(x)(x)=-\frac{9}{2 g} \frac{1}{x^{3}},
$$

is subdominant to the leading nonperturbative correction (53). Hence, the nonperturbative correction (53) being of order $O(1 / x)$ provides asymptotic behavior intermediate to the first and second perturbative corrections being of "alien" nature for semiclassical perturbation theory. It can be used to calculate the leading nonperturbative instanton contribution 
to the energy gap $E_{\mathrm{NPT}, 0}$ as a coefficient in front of the $1 / x$ term in the asymptotic expansion of phase.

Following the philosophy of construction of the approximate wave function for double-well potential $[17,18]$ neither leading nonperturbative correction $\phi_{\mathrm{NPT}, 0}(x)$ nor the perturbative correction $\phi_{\mathrm{PT} 2,0}(x)$ is of importance.

\section{CONNECTION TO PATH INTEGRALS}

Now, when the perturbative and nonperturbative corrections to the phase of the wave function in semiclassical perturbation theory are found, we would like to return to the original issue indicated in the Introduction: the contributions of the flucton and flucton-plus-instanton classical path contributions should naturally appear additively in the path integral for the density matrix $\rho\left(x_{0}\right)=\psi\left(x_{0}\right)^{2}=$ $\exp \left(-2 \phi\left(x_{0}\right)\right)$. In order to do it, the representation (3) used to construct transseries expansion should be rewritten as the product of two factors:

$$
\Psi=e^{-\phi_{\mathrm{PT}}-\phi_{\mathrm{NPT}}}=e^{-\phi_{\mathrm{PT}}} e^{-\phi_{\mathrm{NPT}}} .
$$

As we already know $[2,3]$, the Taylor expansion

$$
\phi_{\mathrm{PT}}(u)=\sum_{n=0} g^{2 n} \phi_{\mathrm{PT}, n}(u)
$$

corresponds to the loop expansion in flucton calculus: $\phi_{\mathrm{PT}, 0}$ is the classical flucton action; one-loop contribution $g^{2} \phi_{\mathrm{PT}, 1}=\log D$ is the logarithm of the determinant; $\phi_{\mathrm{PT}, 2}$ is the two-loop contribution; and, in general, $\phi_{\mathrm{PT}, n}$ is the $n$-loop contribution. It allows us to rewrite the perturbative part of the flucton density $\left(\Psi_{\mathrm{PT}}\right)^{2}$ as the saddle-point expansion,

$e^{-2 \phi_{\mathrm{PT}}}=e^{-2 \phi_{\mathrm{PT}, 0}} F_{0} \equiv \frac{1}{D^{2}} e^{-2 \phi_{\mathrm{PT}, 0}}\left(1-2 g^{4} \phi_{\mathrm{PT}, 2}+\ldots\right)$.

The second factor $e^{-2 \phi_{\mathrm{NPT}}}$ can be expanded in the Taylor series in powers of the nonperturbative phase $\phi_{\mathrm{NPT}}$. It corresponds to the expansion in powers of the exponential in the one-instanton classical action,

$$
e^{-2 \phi_{\mathrm{NPT}}}=1+e^{-S_{0}} F_{1}(x, g)+e^{-2 S_{0}} F_{2}(x, g)+\ldots,
$$

where for functions $F_{1,2, \ldots}$ the first terms in the expansion in powers $g$ can be found explicitly. In particular,

$$
\begin{aligned}
F_{1}= & \int \frac{d x}{g x(1-g x) z_{0}} \\
& \times\left(1-\frac{g^{2}}{2}\left(\frac{83}{6}+\frac{1+2 g x}{(g x)^{2}(1-g x)^{2} z_{0}}\right)+\ldots\right) .
\end{aligned}
$$

Thus, the expansion (58) appears as the expansion in powers $e^{-S_{0}}$. Combining (57) and (58), we arrive at the expansion for density in the form a superposition of saddlepoint contributions (and expansion around each of them multiplied by the product of determinants),

$$
\begin{aligned}
e^{-2 \phi}= & e^{-2 \phi_{\mathrm{PT}, 0}} F_{0}+e^{-2 \phi_{\mathrm{PT}, 0}-S_{0}} F_{0} F_{1} \\
& +\cdots+e^{-2 \phi_{\mathrm{PT}, 0}-n S_{0}} F_{0} \tilde{F}_{n}+\ldots,
\end{aligned}
$$

where $\tilde{F}_{n}$ is a polynomial in $F$ 's. The first term corresponds to the flucton classical trajectory with classical action $\left(2 \phi_{\mathrm{PT}, 0}\right)$, while $\lim _{g \rightarrow 0} \frac{1}{g^{2}} F_{0}(u, g)$ represents the determinant (quadratic fluctuations); the second one is the flucton + instanton trajectory contribution with classical action $\left(2 \phi_{\mathrm{PT}, 0}+S_{0}\right)$, while $\lim _{g \rightarrow 0} \frac{1}{g^{4}} F_{0}(u, g) F_{1}(u, g)$ represents the determinant (quadratic fluctuations) around this trajectory; the $(n+1)$ th term should correspond to the flucton $+n$-instanton contribution with classical action $\left(2 \phi_{\mathrm{PT}, 0}+n S_{0}\right)$, while $F_{0}(u, g=0) \tilde{F}_{n}(u, g=0)$ represents determinant (quadratic fluctuations) around this trajectory; etc. The main point is that different classical paths lead to additive contributions to the path integral, and thus to the density matrix. It is evident that the expansion (59) is different for large positive and negative $x$. The symmetry is restored when the new variable is introduced $\tilde{x}=x-\frac{1}{2 g}$; the expansions become the same.

Furthermore, more close focus on the obtained result reveals one more interesting phenomenon: the interaction between classical objects, which leads to logarithmic terms in the transseries. Indeed, let us look again at the lowest order perturbative and nonperturbative results we already obtained above. The equation reads

$$
u^{2}(1-u)^{2}\left(z_{0}+z_{n p}\right)=\frac{A_{0}}{g} \exp \left(-S_{i}\right)+\cdots
$$

Using the definitions of $z$ and $u$, it means that

$$
\begin{aligned}
-\frac{\psi_{x}^{\prime}(x)}{\psi(x)}= & x(1-g x) z(u)=x(1-g x) \\
& +\frac{1}{x(1-g x)} \frac{A_{0}}{g^{3}} \exp \left(-S_{i}\right)+\cdots
\end{aligned}
$$

Integrating over the coordinate to recover the wave function, one finds

$$
\begin{aligned}
\psi(x) \sim & \exp \left(-\int_{x_{\min }}^{x} d x^{\prime}\left[x^{\prime}\left(1-g x^{\prime}\right)\right.\right. \\
& \left.\left.+\frac{1}{x^{\prime}\left(1-g x^{\prime}\right)} \frac{A_{0}}{g^{3}} \exp \left(-S_{i}\right)\right]\right) \\
\approx & \exp \left(-S_{f}\left(x_{0}\right)\right)\left[1+\log \left(\frac{x\left(1-g x_{\min }\right)}{x_{\min }(1-g x)}\right) \frac{A_{0}}{g^{3}}\right. \\
& \left.\times \exp \left(-S_{i}\right)+\cdots\right],
\end{aligned}
$$


where $x>x_{\min }$, which is some normalization point. While the flucton and instanton actions in the second term appear in the exponent as a sum, the preexponent has nontrivial logarithmic dependence on $x$ and $g$. So, the classical flucton and instanton actions are additive, but the determinants do not simply factorize but indicate instead the appearance of new series with logs.

In the language of paths, this dependence comes from the fact that there is not just one single " $\mathrm{f}+\mathrm{i}$ " trajectory but a whole family of such paths, parametrized by the time $\Delta \tau$ between their centers. Integration over all paths of the family, over $\Delta \tau$, is the source of the discussed interaction. Unfortunately, it is not so simple to calculate explicitly its effect in the path integral formalism. But we do not have to do so; we have already found the total contribution of the " $\mathrm{f}+\mathrm{i}$ " family of paths.

We therefore reached the main goal of the paper: we indeed see additive contributions to the density matrix of the two paths sketched in Fig. 1, the flucton one and the flucton-plus-instanton one. One can find in the exponent the simple sum of both actions; this indicates that generically the flucton and instanton parts of the path are far away and classically do not interact. However, the preexponent does depend on $x$, so at one-loop level, such interaction between them does exist. Note that the integral produces logarithms, of similar origin as interinstanton logarithms in the transseries for the energy. The relative normalization of the two (or more, with multi-instantons) contributions is therefore established.

Finally, we remind the reader that our ultimate goal is to use the semiclassical theory of fluctons and instantons in the QFT settings, in which the same issue of relative normalization is present, but there is no handy generalized Bloch equation available.

\section{ACKNOWLEDGMENTS}

E. S. thanks G. V. Dunne for indicating the work [1]. The work of E. S. is supported in part by the U.S. Department of Energy, Office of Science under Contract No. DE-FG88ER40388. A. V. T. gratefully acknowledges support from the Simons Center for Geometry and Physics, Stony Brook University, at which the research for this paper was initiated and eventually completed.
[1] L. Schulman, Phys. Rev. 176, 1558 (1968).

[2] M. A. Escobar-Ruiz, E. Shuryak, and A. V. Turbiner, Phys. Rev. D 93, 105039 (2016).

[3] M. A. Escobar-Ruiz, E. Shuryak, and A. V. Turbiner, Phys. Rev. D 96045005 (2017).

[4] M. A. Escobar-Ruiz, E. Shuryak, and A. V. Turbiner, Phys. Rev. D 92, 025046 (2015); 92, 089902(E) (2015).

[5] M. A. Escobar-Ruiz, E. Shuryak, and A. V. Turbiner, Phys. Rev. D 92, 025047 (2015).

[6] A. M. Polyakov, Nucl. Phys. B120, 429 (1977).

[7] M. A. Shifman, Pis'ma Zh. Eksp. Teor. Fiz. 147, 444 (2015) [JETP Lett. 120, 386398 (2015)].

[8] L. D. Landau and E. M. Lifshitz, Quantum Mechanics, Non-Relativistic Theory, Course of Theoretical Physics, 3rd ed. (Pergamon, Oxford, 1977), Vol. 3.

[9] J. Zinn-Justin, Nucl. Phys. B192, 125 (1981); B218, 333 (1983).
[10] J. Zinn-Justin and U. D. Jentschura, Ann. Phys. (Amsterdam) 313, 197 (2004); 313, 269 (2004); Phys. Lett. B 596, 138 (2004).

[11] G. V. Dunne and M. Ünsal, Phys. Rev. D 89, 105009 (2014).

[12] In folklore, it is known as the E. M. Lifschitz prescription. Taking, for instance, $\Psi(x)=e^{-g^{2} x^{2}}$, the energy gap can be evaluated up to the multiplicative constant.

[13] E. B. Bogomolny, Phys. Lett. B 91, 431 (1980).

[14] In standard calculations of the exponentially small terms, the logarithmic terms appear in the form $\log \left(-\frac{2}{\lambda}\right)$, see Refs. $[9,10]$, where $\lambda$ is the coupling constant. The meaning of the Bogomolny mechanism in superficial terms is in the replacement of $\log \left(-\frac{2}{\lambda}\right)$ by $-\frac{1}{2} \log \left(\frac{\lambda^{2}}{4}\right)+$ const.

[15] A. V. Turbiner, Usp. Fiz. Nauk 144, 35 (1984) [Sov. Phys. Usp. 27, 668 (1984)].

[16] F. Wöhler and E. Shuryak, Phys. Lett. B 333, 467 (1994).

[17] A. V. Turbiner, Lett. Math. Phys. 74, 169 (2005).

[18] A. V. Turbiner, Int. J. Mod. Phys. A 25, 647 (2010). 